\title{
Management of vascular causes of pulsatile tinnitus
}

\author{
Kazim H Narsinh (ㄷ, , Ferdinand Hui (1) , ${ }^{2}$ Madhavi Duvvuri, ${ }^{3}$ Karl Meisel, ${ }^{4}$ \\ Matthew R Amans (i) ${ }^{3}$
}

\begin{abstract}
- Additional supplemental material is published online only. To view, please visit the journal online (http://dx.doi. org/10.1136/neurintsurg2021-018015)
\end{abstract}

${ }^{1}$ Neurointerventional Radiology, University of California San Francisco, San Francisco,

California, USA

${ }^{2}$ Neurointerventional Radiology, Johns Hopkins University, Baltimore, Maryland, USA ${ }^{3}$ Radiology and Biomedical Imaging, University of California San Francisco, San Francisco,

California, USA

${ }^{4}$ Neurology, University of California San Francisco, San

Francisco, California, USA

\section{Correspondence to} Dr Kazim H Narsinh, University of California San Francisco, San Francisco, California, USA; kazim.narsinh@ucsf.edu

Received 14 July 2021

Accepted 23 January 2022

Check for updates

(c) Author(s) (or their employer(s)) 2022. No commercial re-use. See rights and permissions. Published by BMJ.

To cite: Narsinh KH, Hui F, Duvvuri $\mathrm{M}_{\text {, et al. }}$

J Neurolntervent Surg Epub ahead of print: [please include Day Month Year]. doi:10.1136/

neurintsurg-2021-018015

\section{ABSTRACT}

Pulsatile tinnitus is a debilitating symptom affecting millions of Americans and can be a harbinger of hemorrhagic or ischemic stroke. Careful diagnostic evaluation of pulsatile tinnitus is critical in providing optimal care and guiding the appropriate treatment strategy. When a vascular cause of pulsatile tinnitus has been established, attention must be focused on the patient's risk of hemorrhagic stroke, ischemic stroke, or blindness, as well as the risks of the available treatment options, in order to guide decision-making. Herein we review our approach to management of the vascular causes of pulsatile tinnitus and provide a literature review while highlighting gaps in our current knowledge and evidence basis.

\section{INTRODUCTION}

Pulsatile tinnitus (PT) is a symptom referring to an abnormal perception of rhythmic sound without an extracorporeal source that impacts between 3 and 5 million Americans. ${ }^{1-4}$ PT can have a tremendous impact on patients' psychological and physical health, leading to insomnia, anxiety, depression, and poor concentration. ${ }^{5}$ Seeking an underlying cause of PT is essential because many of them pose a significant risk of hemorrhagic stroke, ischemic stroke, or blindness to the patient. Hence some of the causes of PT warrant treatment to mitigate risk of stroke or blindness, while other causes may be treated to address the symptom itself and its psychiatric comorbidities. Advances in neuroimaging and endovascular treatment have resulted in increased detection of vascular causes ${ }^{67}$ and therapeutic options have burgeoned. Once a vascular etiology has been established, treatment recommendations should be based on the natural history of the disease, treatment risks, and the efficacy of treatment. This article will review the literature related to the management of vascular causes of PT, address gaps in knowledge and evidence, and provide a blueprint for future studies.

\section{METHODS}

\section{Literature search strategy and selection criteria}

For this narrative review, a literature search was performed by the authors of the PubMed and PMC databases, for peer-reviewed studies published from 1975 to 2021 in the English language using the following key words: 'pulsatile tinnitus' (Title) + '(cause of pulsatile tinnitus)' + 'management' or 'treatment'. For 'cause of pulsatile tinnitus', keywords included: 'atherosclerotic carotid artery disease', 'intracranial arterial aneurysms', 'arteriovenous malformations', 'arteriovenous fistulas', 'dural arteriovenous fistulas', 'fistula', 'internal carotid artery', 'idiopathic intracranial hypertension', 'venous abnormalities', 'jugular bulb abnormality', 'sigmoid sinus abnormality', 'sigmoid sinus dehiscence', 'emissary vein', 'glomus tumor', 'superior semicircular canal dehiscence', and 'metabolic cause' and 'systemic cause'. In addition, we performed a reverse bibliography search from previously published systematic reviews or meta-analyses. Each study was critically reviewed. Duplicates generated across multiple searches were excluded. Articles were included if pulsatile tinnitus was referenced during the pre-interventional or post-interventional period. Studies that did not specify the interventional technique or outcome of the procedure were excluded. Studies were excluded if subsequent papers looked at outcomes from the same patient group. Non-full text articles were excluded. The PRISMA (Preferred Reporting Items for Systematic Reviews and Meta-Analyses) flow diagram is shown in online supplemental figure 1).

\section{Data extraction}

Each study was analyzed by two independent reviewers (MD, KHN) to assess clinical outcomes, including improvement or resolution of symptoms (ie, clinical success), rate of complication (ie, technical success), and the limitations of the work. In cases of disagreement between the two reviewers, a third reviewer (MRA) served as adjudicator.

\section{MANAGEMENT OF VASCULAR CAUSES OF PT Venous}

Among vascular causes of PT, a venous etiology can often be distinguished from an arterial etiology on the basis of history and physical examination. Venous PT is described as a lower pitched 'whooshing' sound that can be alleviated by neck maneuvers. In particular, compression of the ipsilateral internal jugular vein (IJV) or suboccipital venous plexus can alleviate symptoms, while compression of the contralateral IJV can exacerbate symptoms. When a venous cause of PT is suspected, we find cerebral venous manometry and balloon test occlusion to be a critical part of the diagnostic evaluation that guides subsequent management (see UCSF Cerebral Venous Disorder Testing form included as online supplemental material). This detailed evaluation is typically performed with the patient awake in order to accurately assess intracranial venous pressures and subjective PT. 
Idiopathic intracranial hypertension

A common venous cause of PT is idiopathic intracranial hypertension (IIH). IIH has an incidence of 20 per 100000 overweight women of childbearing age, with increasing prevalence due to the obesity epidemic. Multiple medications and substances have been linked to IIH, with the most evidence available for an association between IIH and hypervitaminosis A, tetracyclines, and growth hormone. Stopping these medications may result in resolution of IIH symptoms. For most IIH patients, first-line treatment consists of weight loss and acetazolamide. ${ }^{8}$ Acetazolamide is a carbonic anhydrase inhibitor that reduces the rate of cerebrospinal fluid (CSF) production. The NORDIC (Neuro-Ophthalmology Research Disease Investigator Consortium) trial was a multicenter, randomized, double-masked, placebo-controlled study of acetazolamide with a low-sodium weight-reduction diet versus a low-sodium weight-reduction diet alone in 165 patients with IIH meeting the modified Dandy criteria and having mild visual loss. ${ }^{8}$ The trial found that perimetric mean deviation (a measure of global visual field loss), papilledema grade, and CSF opening pressure was improved in patients taking acetazolamide versus controls at 6 months follow-up. Based on these results, first-line treatment for IIH consists of a low-sodium weight-reduction diet and acetazolamide dose-escalation until $2-4 \mathrm{~g}$ per day are tolerated. Relative contraindications to acetazolamide include sulfa allergy and pregnancy. Acetazolamide has multiple side effects that can make medication adherence difficult. These include, for example, oral and digital paresthesias, malaise, metallic taste, fatigue, nausea, vomiting, metabolic acidosis, and nephrolithiasis. Topiramate also inhibits carbonic anhydrase activity, and is effective in the treatment of migraine headache and facilitating weight loss. These features have made topirimate a potential alternative therapeutic option in IIH, although data supporting its efficacy for visual field improvement are limited to case series.

IIH patients who have progressive visual loss or headache, and have failed, are intolerant to, or are non-compliant with medical therapy, may benefit from operative intervention. Operations include optic nerve sheath fenestration (ONSF), CSF diversion, bariatric surgery, and venous sinus stenting.

After ONSF, the majority of patients have improved papilledema and visual fields. However, approximately $20 \%$ of ONSF patients will have deterioration after initial improvement, and most of these patients will go on to need additional surgery, typically either additional ONSF or CSF diversion. ${ }^{9} 10$ Major vascular complications are most likely to occur during the second ONSF. ${ }^{10}$ In most series, the complication rate from ONSF is high, ranging from $20-40 \% .{ }^{10} 11$ The most common complications are ocular motility disorders (eg, diplopia due to cranial nerve palsy) or pupillary dysfunction (eg, anisocoria) that are often transient; however, more serious complications, such as central retinal artery occlusions, orbital hematomas, orbital apex syndrome, orbital cellulitis, optic disc hemorrhage, or traumatic optic neuropathy, also occur.

After CSF diversion for IIH, most patients have improved headache, papilledema, and/or visual acuity. However, CSF diversion has a very high rate of revision surgery of up to $43 \%$, usually due to shunt obstruction/failure or low-pressure headache. ${ }^{11} 12$ In addition, CSF diversion has a high complication rate of up to $33 \%$, including shunt infection, subdural hematoma, and CSF leak. ${ }^{11} 12$

Bariatric surgery, such as Roux-en-Y gastric bypass, sleeve gastrectomy, or gastric banding, has also been proposed as a treatment for IIH because of the strong association between obesity and IIH. In a randomized controlled trial, bariatric surgeries resulted in decreased CSF pressures and increased weight loss compared with a community weight management intervention (eg, Weight Watchers). ${ }^{13}$ However, there were no significant differences in visual function (ie, perimetric mean deviation or papilledema grade), headache scores, or IIH symptoms between the two groups. In addition, nearly $40 \%$ of patients in the bariatric surgery arm suffered an adverse event during the 24 month follow-up period, which included hospitalization for IIH exacerbation in $18 \%$ of bariatric surgery patients during the first postoperative year. Only one patient $(3 \%)$ in the bariatic surgery arm underwent reoperation for a bowel obstruction complication; none of the bariatric surgery patients underwent subsequent CSF diversion. In earlier series, complication rates of bariatric surgery were as high as $55 \% .^{11}$

In comparison to the aforementioned surgeries, venous sinus stenting (VSS) has a relatively favorable complication rate of $6.6 \%$, with a major complication rate (which includes subdural hemorrhage, sinus or stent thrombosis, and retroperitoneal hemorrhage) of $2.3 \% .^{14} 15$ Relapse rates after VSS, however, can vary between $10-26 \% .{ }^{16}$ In spite of this, because of the relatively low complication rate, VSS offers a favorable risk-tobenefit ratio compared with optic nerve sheath fenestration, CSF diversion, or bariatric surgery for medically refractory cases of IIH with worsening papilledema and/or vision loss (figure 1).

Apart from IIH, other causes of turbulent flow in the transverse or sigmoid sinus, emissary veins, condylar veins, or internal jugular vein can cause PT. ${ }^{18-20}$ Various endovascular treatment strategies have been described to address these abnormalities (online supplemental table 1) with resolution of symptoms reported in most patients; however, these data are limited to case reports and small series and therefore strong conclusions are difficult to make in light of publication bias. These venous causes of PT can be separated into abnormalities of the dural venous sinuses, the jugular vein, or the emissary/condylar veins.

\section{Dural venous sinus abnormalities}

Stenosis of the transverse or sigmoid sinus is defined by a transstenotic pressure gradient and can be found without signs or symptoms of elevated intracranial pressure. Stenosis at the proximal transverse/sigmoid sinus junction is the most common location, and can be caused by chronic sinus thrombosis or arachnoid granulations. The stenosis causes altered hemodynamics and turbulence that reverberates through the temporal bone to auditory structures. ${ }^{21}$ Venous sinus stenting has been used to alleviate symptoms in small series and case reports with acceptable morbidity. ${ }^{22}$

Sigmoid sinus wall abnormalities that can cause PT include sigmoid sinus diverticula and sigmoid plate dehiscence or thinning. Coil embolization of sigmoid sinus diverticula is generally well-tolerated and leads to resolution of symptoms in most small series (online supplemental table 1). A case of sigmoid sinus diverticulum is presented in figure 2 . For cases of sigmoid sinus diverticula, we strongly suggest evaluating for IIH as a potential cause of the sigmoid sinus diverticula. For cases in which sigmoid sinus diverticula coexist with IIH, treatment of the IIH with VSS can cure PT, while isolated coil embolization of the diverticula without treatment of the underlying IIH can lead to symptom recurrence. Sigmoid sinus cortical plate dehiscence has been treated with surgical reconstruction of the sinus wall with resolution of symptoms in $74 \%$ of a small retrospective series of patients. Complications from sigmoid sinus resurfacing, including CSF leak or sinus thrombosis, are reported in up to $24 \%$ of patients, with major complications needing pharmacologic or surgical interventions reported in 6\% of patients. ${ }^{23} 24$ However, comparison with endovascular techniques such as VSS 

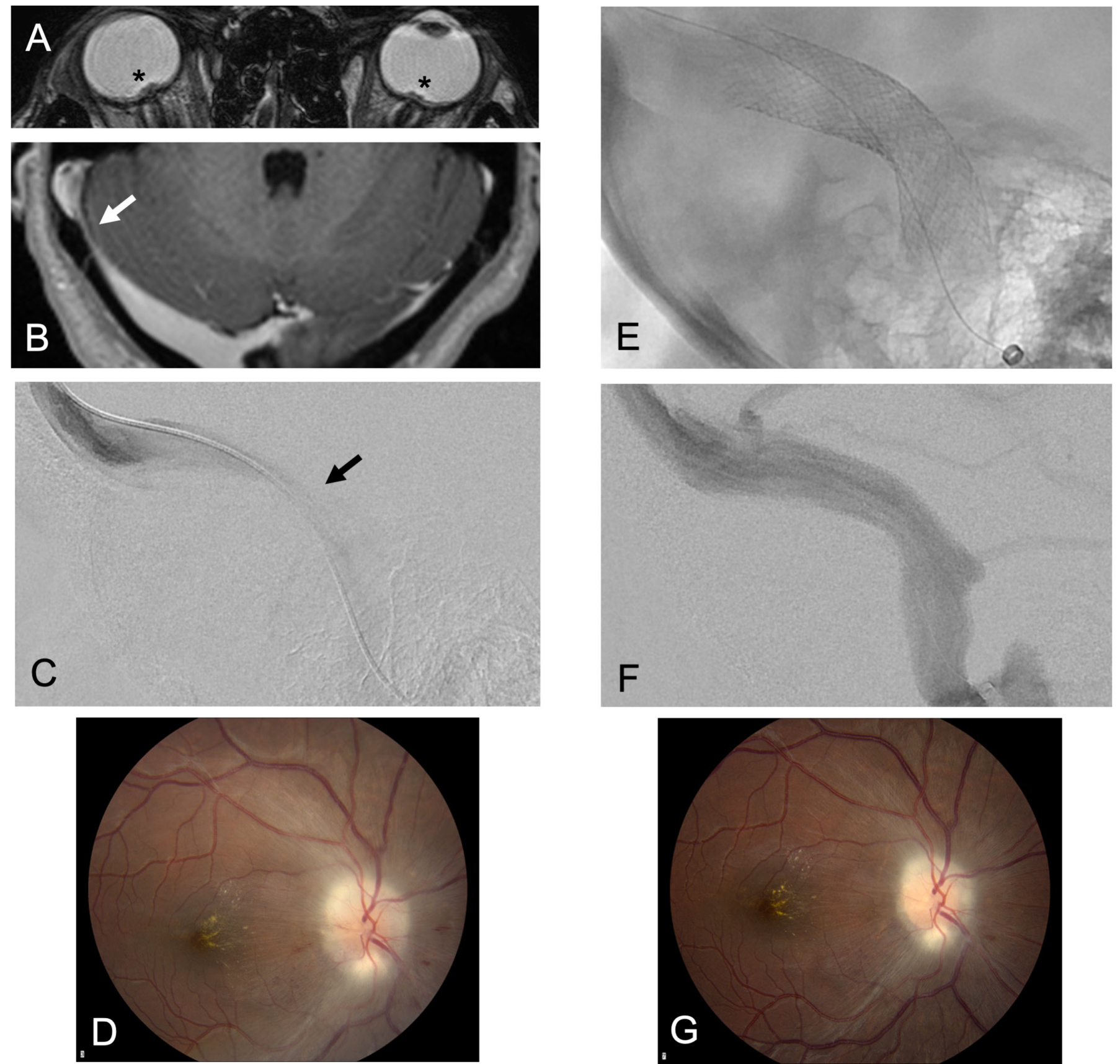

Figure 1 Idiopathic intracranial hypertension. A young woman presented with right pulse-synchronous pulsatile tinnitus, worse when laying down, exacerbated by right neck compression, with positional headaches and vision changes. (A) Axial balanced steady-state-free-precession (bSSFP; FIESTA) MRI demonstrates optic disc protrusion (asterisk). (B) Axial T1-weighted contrast-enhanced gradient-echo (FSPGR) demonstrates right transverse-sigmoid sinus junction stenosis (arrow). Lumbar puncture opening pressure was $>60 \mathrm{~cm}$ of water and she had decreased visual acuity on the right (20/100). Venous manometry gradient was $18 \mathrm{~mm} \mathrm{Hg}$. (C) Right transverse sinus venogram in lateral projection demonstrates stenosis (arrow). (D) Fundoscopy demonstrates Frisen grade 2 papilledema. (E) Venous sinus stenting of the right transverse-sigmoid sinus in lateral projection. (F) Cerebral angiogram in venous phase and lateral projection demonstrates no residual stenosis of the right transverse-sigmoid sinus. One week after stenting, visual acuity improved to $20 / 25$ and (G) fundoscopy demonstrates improved papilledema to Frisen grade 1 . At 3 months, the lumbar puncture opening pressure was $11 \mathrm{~cm}$ of water and at 5 months, visual acuity had improved to 20/20 with no residual papilledema.

or coil embolization has not yet been performed. ${ }^{1825-28}$ Of note, if the sigmoid sinus dehiscence is due to underlying IIH, resurfacing does not address the underlying disease process, while VSS can be curative.

\section{Emissary vein anomalies}

Emissary veins connect the intracranial and extracranial venous systems and are characterized by a thin wall and a valveless structure. Three emissary veins are most commonly implicated in PT: the posterior condylar vein, the mastoid emissary vein, and the petrosquamosal vein. Turbulent flow through these emissary veins can cause PT, particularly in the setting of intracranial venous system obstruction or an arteriovenous shunt. ${ }^{29}{ }^{30}$ In the absence of an arteriovenous shunt, determining whether emissary vein flow contributes to a patient's PT can be challenging, and we find balloon test occlusion to be very helpful in these 

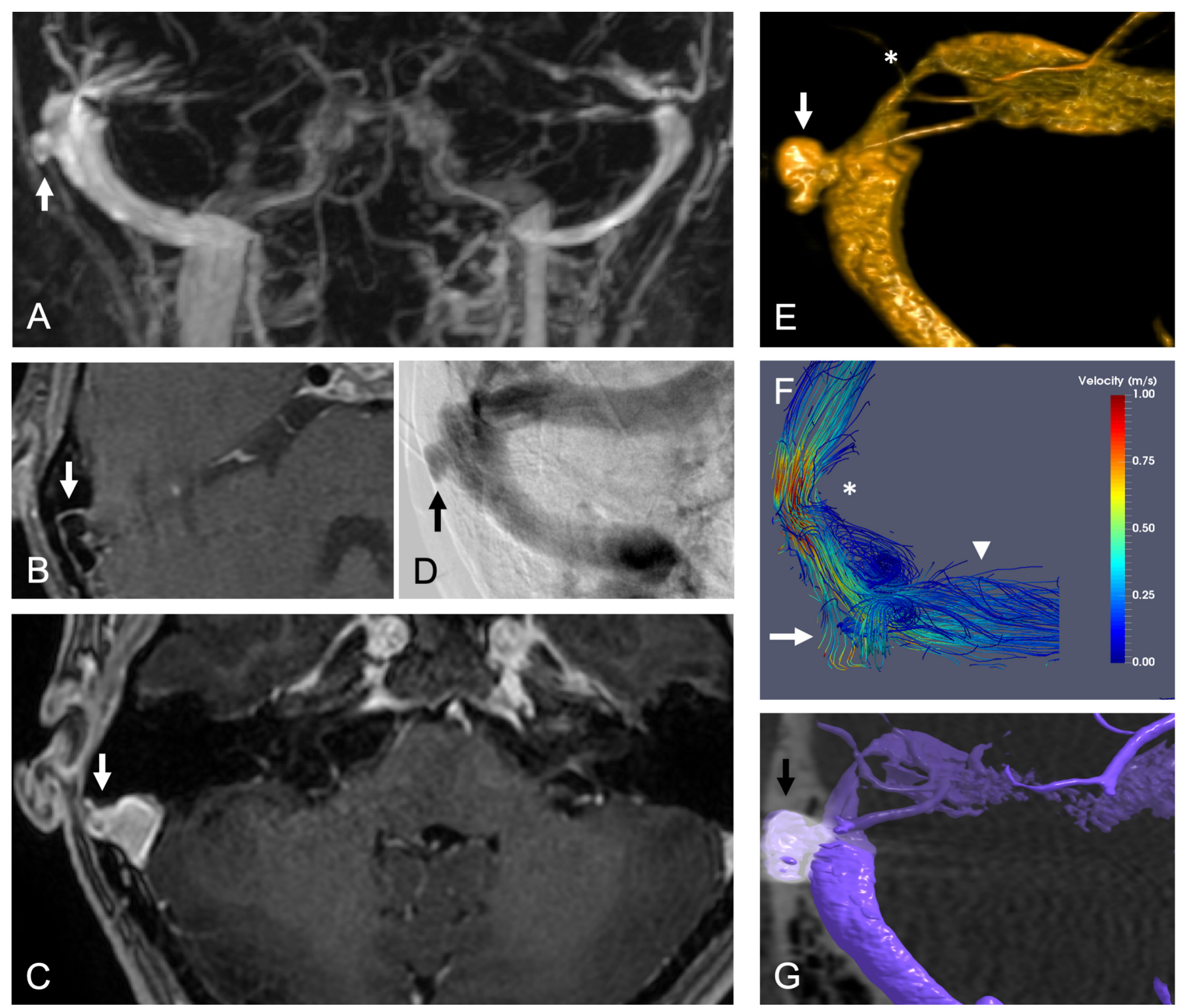

Figure 2 Sigmoid sinus diverticulum. A middle-aged woman presented with right-sided pulsatile tinnitus that was exacerbated by left neck compression and improved with right neck compression. Her lumbar puncture opening pressure was normal. (A) Coronal maximum intensity projection (MIP) reformat of time-resolved contrast-enhanced (TRICKS) MRA demonstrates right sigmoid sinus diverticulum (arrow). (B) Axial T1weighted contrast-enhanced spin-echo (CUBE) MRI demonstrates signal void (arrow) due to flow in right sigmoid sinus diverticulum. (C) Axial T1-weighted contrast-enhanced gradient-echo (FSPGR) MRI demonstrates enhancement (arrow) of diverticulum contiguous with the right sigmoid sinus. (D) Left internal carotid arteriogram in venous phase and Townes projection demonstrates right sigmoid sinus diverticulum (arrow). (E) Volume rendering of 3D rotational cone beam $\mathrm{CT}$ in venous phase demonstrates sigmoid sinus diverticulum (arrow) and upstream venous stenosis (asterisk). (F) 4D flow MRI fluid dynamic modeling with streamlines demonstrates elevated velocity at the upstream venous sinus stenosis (asterisk), flow into the sigmoid sinus diverticulum (arrow), and vortex in the downstream sigmoid sinus (arrowhead). Reproduced with permission from Amans et al. ${ }^{28}$ (G) Coil embolization of sigmoid sinus diverticulum (arrow) resulted in resolution of symptoms and abnormal 4D flow characteristics. 3D, threedimensional; 4D, four-dimensional; MRA, MR angiography.

circumstances. Coil embolization of these emissary veins to treat PT has been described in small case series with good results and no morbidity. ${ }^{203132}$

\section{Jugular vein anomalies}

Jugular vein anomalies are present in up to $10-15 \%$ of normal subjects and include high-riding jugular bulb and jugular dehiscence/diverticulum. When the jugular bulb lies abnormally at the level of the hypotympanum or more superiorly, and the jugular plate is thin, turbulent flow adjacent to the mastoid air cells and cochlea can produce venous PT. Stenosis of the internal jugular vein, due to thrombosis or compression by the styloid process, can exacerbate symptoms, particularly when the contralateral internal jugular vein is atretric. Surgical treatment such as jugular vein ligation or lowering of the jugular bulb is associated with high complication and low efficacy rates. ${ }^{33} 34$ Endovascular treatment, such as coiling, stent-assisted coiling, or even WEB (Woven EndoBridge) embolization, have been reported as effective in case reports and small case series (online supplemental table 1). However, jugular vein stenting is generally inadvisable because of risk of stent migration, stent thrombosis, or lower cranial neuropathy. 


\section{Arterial}

Arterial causes of PT include carotid artery stenosis, vertebral or carotid dissection, fibromuscular dysplasia, aneurysm, aberrant internal carotid artery, and dural arteriovenous fistula (dAVF). Treatment of the arterial causes of PT usually result in resolution of symptoms, but evidence is limited to small case series and reports (online supplemental table 1). Often, indications for treatment of the arterial causes of PT include not only the disabling symptom itself, but prevention of ischemic or hemorrhagic stroke, which form a stronger evidence basis.

A comprehensive discussion of myriad dAVFs and their preferred treatment method are beyond the scope of this review. However, some general principles regarding management of dAVF should guide treatment. First, the primary clinical goal for dAVF treatment remains reduction of the risk of future hemorrhage. Therefore, resolving cortical venous reflux takes precedence over curing PT. For low-risk dAVF (ie, without cortical venous reflux), a detailed discussion with the patient is needed to ascertain the level of disability related to PT (for example, using the Tinnitus Handicap Inventory ${ }^{35}$ ), which then guides discussion of the risks of any treatment offered to reduce that disability. If the dAVF is low-risk and symptoms are tolerable, conservative management is certainly a reasonable strategy (figure 3). Some patients may be concerned about the risk of a low-risk dAVF progressing to a higher-risk dAVF. While this is quite rare, non-invasive imaging is currently unable to reliably detect high-risk dAVF features, particularly when subtle. As such, conservative management requires detailed patient counseling regarding symptomatology. Specifically, resolution of PT in a dAVF patient may indicate disease progression rather than

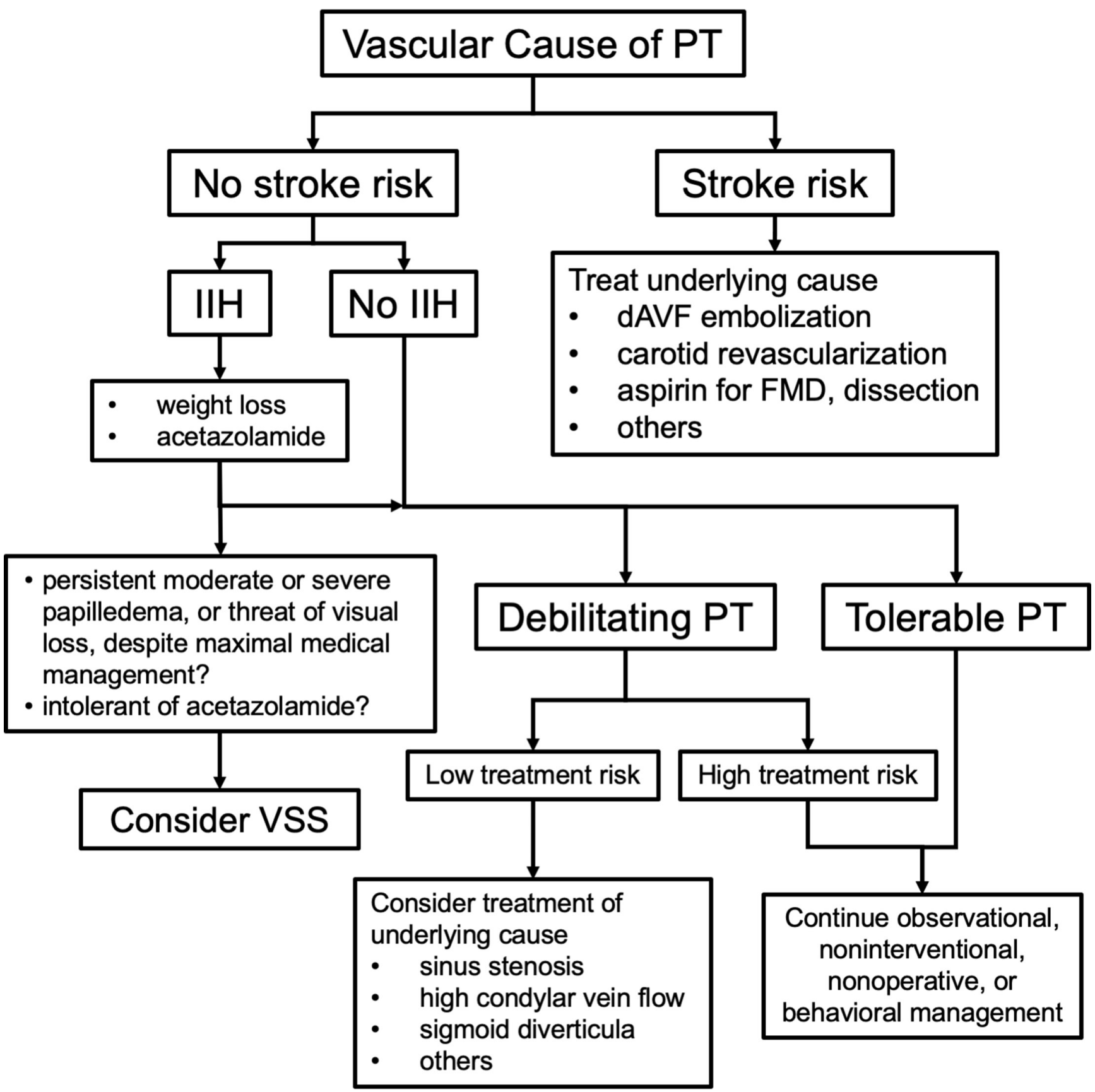

Figure 3 Suggested algorithm for the treatment approach to a patient with a suspected vascular cause of pulsatile tinnitus. dAVF, dural arteriovenous fistula; FMD, fibromuscular dysplasia; IIH, idiopathic intracranial hypertension; PT, pulsatile tinnitus; VSS, venous sinus stent; . 

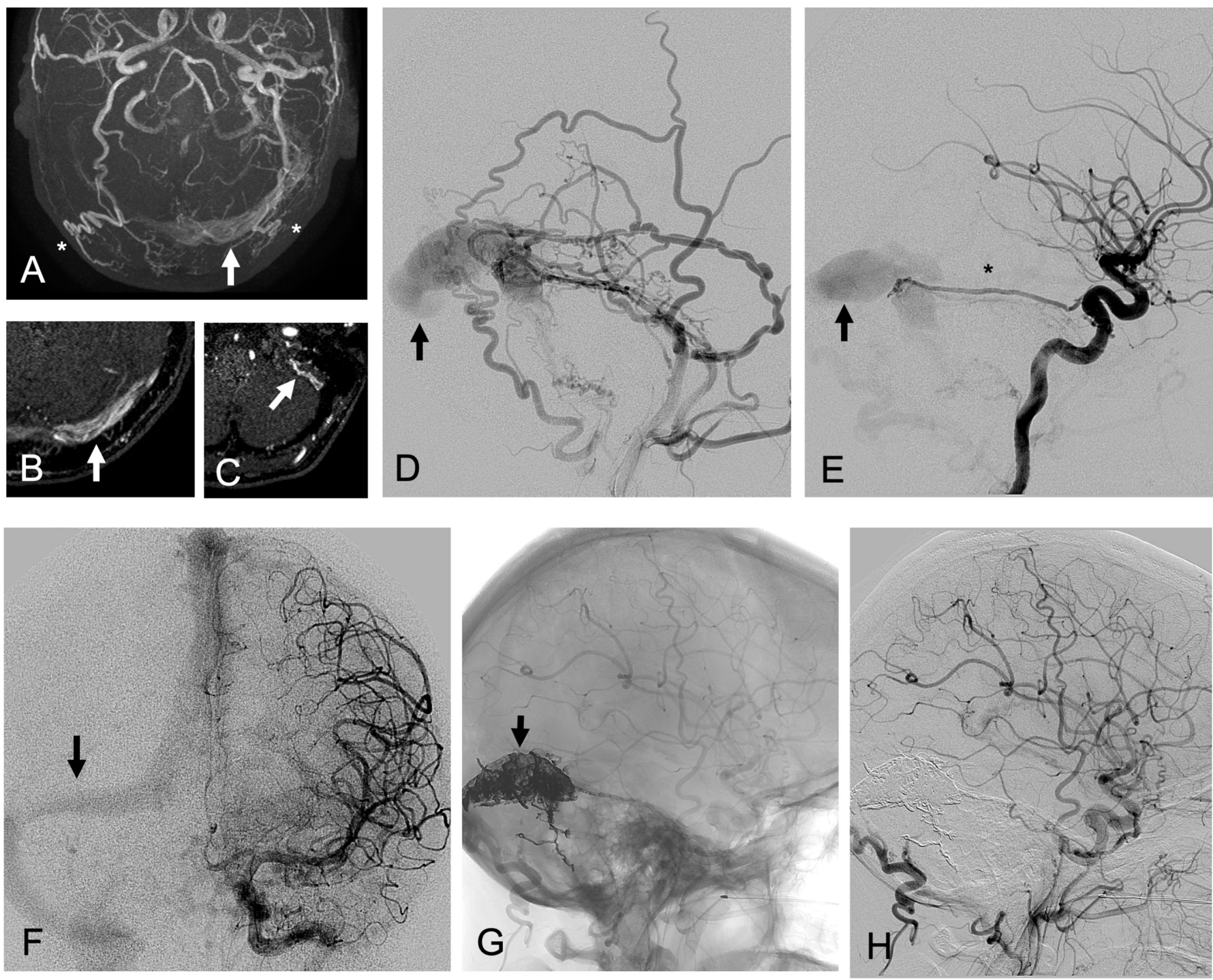

Figure 4 Left transverse-sigmoid sinus dural arteriovenous fistula. A middle-aged woman presented with left pulsatile tinnitus that resolved 1 week previously, and has now developed headache, nausea, and incoordination. (A) Axial maximum intensity projection (MIP) of time-of-flight (TOF) MRA demonstrates enlarged occipital arteries (asterisks) and flow-related enhancement in the left transverse (arrow) and sigmoid sinuses. (B) Axial TOF MRA demonstrates flow-related enhancement in the left transverse sinus (arrow) and enlarged bilateral occipital arteries. (C) Axial TOF MRA demonstrates flow-related enhancement in dural arteries of the left sigmoid sinus wall. (D) Left external carotid arteriogram in lateral projection demonstrates arteriovenous shunting into the left transverse sinus by enlarged occipital artery, middle meningeal artery, superficial temporal artery, and ascending pharyngeal artery branches. (E) Left internal carotid arteriogram in lateral projection demonstrates arteriovenous shunting into the left transverse sinus (arrow) by an enlarged lateral tentorial branch (asterisk) of the meningohypophyseal trunk. (F) Venous phase of left internal carotid arteriogram in frontal projection shows right-dominant venous system (arrow=right transverse sinus). Ethylene vinyl alcohol copolymer embolization via a left occipital artery transmastoid branch with intentional occlusion of the sinus (G) resulted in angiographic resolution of arteriovenous shunting on $(\mathrm{H})$ post-embolization left common carotid arteriogram in lateral projection. MRA, MR angiography.

resolution-for instance, if the jugular vein has occluded and venous drainage is diverted retrograde into cortical veins. ${ }^{36} 37$ If dAVF treatment is undertaken, endovascular treatment is typically first-line therapy, as determined by cervicocerebral angiography (figure 4). Transvenous embolization is our preferred approach for low-risk marginal sinus and indirect carotidcavernous dAVFs due to external carotid artery supply to cranial nerves and extensive extracranial-to-intracranial artery anastomoses, as previously detailed. ${ }^{38} 39$ When contemplating the risk of transvenous embolization, we always consider risk of intracranial hemorrhage, venous infarction, or intracranial venous hypertension, based on the venous drainage pattern. For example, if cortical veins are draining into the recipient venous pouch, transvenous embolization should not be performed. ${ }^{40}$

\section{Non-operative treatment of tinnitus}

Our diagnostic approach to a PT patient includes a complete history, physical examination, and imaging evaluation. If, after excluding all 'dangerous' causes of PT and, despite a thorough evaluation, a cause for the patient's tinnitus cannot be found, or the underlying cause cannot be safely or effectively treated surgically or medically, behavioral treatments can be therapeutic. Effective behavioral treatments for tinnitus include tinnitus retraining therapy (TRT), cognitive behavioral therapy (CBT), 
acceptance and commitment therapy, and mindfulness-based stress reduction, ${ }^{41-44}$ with the strongest evidence supporting use of TRT and CBT. However, each treatment provides patients with coping skills that allow the tinnitus to recede into the background so that patients can continue with their lives. TRT focuses on sound habituation, while CBT focuses on dysfunctional beliefs about tinnitus and associated compensation behaviors. Specifically, in a randomized controlled clinical trial of 492 patients in the Netherlands, stepped-care tinnitus management (combining elements of tinnitus retraining therapy within a CBT framework) was more effective than standard care in improving tinnitus severity, impairment, health-related quality of life, negative emotional states, tinnitus-related catastrophic thinking, and tinnitus-related fear for 4 months after treatment ended. Sound generating devices, as an isolated treatment approach, have not been proven as effective. Therefore, an integrated treatment approach which provides sustained psychoeducation and audiologic expertise should be provided rather than fragmented care for tinnitus. Transcranial magnetic stimulation may also have a role in tinnitus suppression, but additional trials are needed to demonstrate long-term reproducible efficacy. ${ }^{45} 46$

\section{FUTURE PERSPECTIVE}

Multiple clinical trials for patients with medically refractory IIH are underway to assess the efficacy of VSS using a variety of different stents with or without comparison to CSF diversion. New stent designs may be needed to reduce the risk of treatment failure after VSS. Endovascular treatment of other venous causes of PT are limited to case reports and series, and it is unlikely that any one center will be able to report a significantly larger case series because of the rarity of these cases and numerous potential anatomic causes. Further study of the other venous causes of PT is also limited by a paucity of objective assessment methods. We have provided our UCSF Cerebral Venous Disorder Testing form as online supplemental material, which guides our assessment of venous causes of PT currently. Development of patient-specific three-dimensional-printed flow models that mimic a patient's hemodynamic conditions also allows physicians to better narrow down the specific causes of a patient's PT, and simulate treatment before the intervention. ${ }^{47}$ In the future, phase-contrast MRI (ie, four-dimensional flow), fluid dynamic modeling with sound simulation, and intravascular sound recordings will likely play a larger role in disease assessment, particularly as part of clinical trials. Arterial causes of PT, such as carotid stenosis and dAVF, can bear a risk of stroke in addition to causing PT, and therefore may warrant treatment for multiple reasons. A suggested algorithm to approach treatment of a patient with a suspected vascular cause of PT is outlined in figure 3.

\section{CONCLUSION}

PT can be a maddening symptom with debilitating psychiatric impact, and has myriad causes, some of which pose significant risk of ischemic or hemorrhagic stroke or possible blindness. Organizing the causes of PT into structural, metabolic, and vascular groups facilitates appropriate testing, referral, and treatment. ${ }^{7}$ After a complete evaluation, if a vascular cause has been established, one must carefully consider the disease's natural history, degree of patient debilitation, goals and risks of treatment, and rationale for the treatment choice. Unfortunately, for many vascular causes of PT, evidence for treatment is mostly limited to case reports and series, with the notable exception of randomized controlled clinical trials for IIH. Clinical trials are needed to establish the role of VSS for IIH.
Contributors KHN wrote the manuscript and supervised the work. MD assisted with the literature review. FH and KM provided critical feedback. MRA provided critical feedback and supervised the work

Funding This study was funded by National Institute of Biomedical Imaging and Bioengineering (5R01EB012031), National Institute of Neurological Disorders and Stroke (U54 NS065705), National Heart, Lung, and Blood Institute (R56HL149124), Congressionally Directed Medical Research Programs (W81XWH-21-1-0753).

Competing interests None declared.

Patient consent for publication Not applicable.

Ethics approval This study does not involve human participants.

Provenance and peer review Commissioned; externally peer reviewed.

Supplemental material This content has been supplied by the author(s). It has not been vetted by BMJ Publishing Group Limited (BMJ) and may not have been peer-reviewed. Any opinions or recommendations discussed are solely those of the author(s) and are not endorsed by BMJ. BMJ disclaims all liability and responsibility arising from any reliance placed on the content. Where the content includes any translated material, BMJ does not warrant the accuracy and reliability of the translations (including but not limited to local regulations, clinical guidelines, terminology, drug names and drug dosages), and is not responsible for any error and/or omissions arising from translation and adaptation or otherwise.

\section{ORCID iDs}

Kazim H Narsinh http://orcid.org/0000-0002-2019-5461

Ferdinand Hui http://orcid.org/0000-0003-3759-7886

Matthew R Amans http://orcid.org/0000-0002-8209-0534

\section{REFERENCES}

1 Heller AJ. Classification and epidemiology of tinnitus. Otolaryngol Clin North Am 2003;36:239-48

2 Bhatt JM, Lin HW, Bhattacharyya N. Prevalence, severity, exposures, and treatment patterns of tinnitus in the United States. JAMA Otolaryngol Head Neck Surg 2016;142:959-65

3 Nondahl DM, Cruickshanks KJ, Wiley TL, et al. Prevalence and 5-year incidence of tinnitus among older adults: the epidemiology of hearing loss study. J Am Acad Audiol 2002:13:323-31.

4 Adams PF, Hendershot GE, Marano MA, et al. Current estimates from the National Health Interview Survey, 1996. Vital Health Stat 10 1999;10:1-203.

5 Salazar JW, Meisel K, Smith ER, et al. Depression in patients with tinnitus: a systematic review. Otolaryngol-Head Neck Surg 2019;161:28-35.

6 Cummins DD, Caton MT, Shah V, et al. MRI and MR angiography evaluation of pulsatile tinnitus: a focused, physiology-based protocol. I Neuroimaging

7 Narsinh KH, Hui F, Saloner D. Diagnostic approach to pulsatile tinnitus: a review. JAMA Otolaryngol - Head Neck Surg 2022

8 Wall M, McDermott MP, NORDIC Idiopathic Intracranial Hypertension Study Group Writing Committee, . et al. Effect of acetazolamide on visual function in patients with idiopathic intracranial hypertension and mild visual loss: the idiopathic intracranial hypertension treatment trial. JAMA 2014;311:1641-51.

9 Fonseca PL, Rigamonti D, Miller NR, et al. Visual outcomes of surgical intervention for pseudotumour cerebri: optic nerve sheath fenestration versus cerebrospinal fluid diversion. Br J Ophthalmol 2014;98:1360-3.

10 Plotnik JL, Kosmorsky GS. Operative complications of optic nerve sheath decompression. Ophthalmology 1993;100:683-90.

11 Kalyvas AV, Hughes M, Koutsarnakis C, et al. Efficacy, complications and cost of surgical interventions for idiopathic intracranial hypertension: a systematic review of the literature. Acta Neurochir 2017;159:33-49.

12 Satti SR, Leishangthem L, Chaudry MI. Meta-analysis of CSF diversion procedures and dural venous sinus stenting in the setting of medically refractory idiopathic intracranial hypertension. AJNR Am J Neuroradiol 2015:36:1899-904.

13 Mollan SP, Mitchell JL, Ottridge RS, et al. Effectiveness of bariatric surgery vs community weight management intervention for the treatment of idiopathic intracranial hypertension: a randomized clinical trial. JAMA Neurol 2021;78:678.

14 Kalyvas A, Neromyliotis E, Koutsarnakis C, et al. A systematic review of surgical treatments of idiopathic intracranial hypertension (IIH). Neurosurg Rev 2021:44:773-92

15 Townsend RK, Jost A, Amans MR, et al. Major complications of dural venous sinus stenting for idiopathic intracranial hypertension: case series and management considerations. J Neurointerv Surg;45:neurintsurg-2021-017361.

16 Kahan J, Sundararajan S, Brown K, et al. Predicting the need for retreatment in venous sinus stenting for idiopathic intracranial hypertension. J Neurointerv Surg 2021;13:574-9.

17 Garner RM, Aldridge JB, Wolfe SQ, et al. Quality of life, need for retreatment, and the re-equilibration phenomenon after venous sinus stenting for idiopathic intracranial hypertension. J Neurointerv Surg 2021;13:79-85.

18 Amans MR, Haraldsson $\mathrm{H}, \mathrm{Kao} \mathrm{E}$, et al. MR venous flow in sigmoid sinus diverticulum. AJNR Am J Neuroradiol 2018;39:2108-13. 
19 Kao E, Kefayati S, Amans MR, et al. Flow patterns in the jugular veins of pulsatile tinnitus patients. J Biomech 2017;52:61-7.

20 Alexander MD, Meisel KM, Halbach VV, et al. Enlarged condylar veins as a source of pulsatile tinnitus: angiographic features and confirmation with venous balloon test occlusion. Neurographics 2017:7:363-8.

21 Pereira VM, Cancelliere NM, Najafi M, et al. Torrents of torment: turbulence as a mechanism of pulsatile tinnitus secondary to venous stenosis revealed by high-fidelity computational fluid dynamics. J Neurointerv Surg 2021;13:732-7.

22 Lenck S, Labeyrie M-A, Vallee F, et al. Stent placement for disabling pulsatile tinnitus caused by a lateral sinus stenosis: a retrospective study. Oper Neurosurg 2017:13:560-5.

23 Liu GS, Boursiquot BC, Blevins NH, et al. Systematic review of temporal boneresurfacing techniques for pulsatile tinnitus associated with vascular wall anomalies. Otolaryngol-Head Neck Surg 2019;160:749-61.

24 Raghavan P, Serulle Y, Gandhi D, et al. Postoperative imaging findings following sigmoid sinus wall reconstruction for pulse synchronous tinnitus. AJNR Am J Neuroradiol 2016;37:136-42.

25 Eisenman DJ, Raghavan P, Hertzano R, et al. Evaluation and treatment of pulsatile tinnitus associated with sigmoid sinus wall anomalies. Laryngoscope 2018;128:S1-13.

26 Houdart E, Chapot R, Merland JJ. Aneurysm of a dural sigmoid sinus: a novel vascular cause of pulsatile tinnitus. Ann Neurol 2000;48:669-71.

27 Lenck S, Mosimann PJ, Labeyrie M-A, et al. Pulsatile tinnitus caused by an aneurysm of the transverse-sigmoid sinus: a new case report and review of literature. J Neuroradiol I Neuroradiol 2012;39:276-9.

28 Amans MR, Stout C, Dowd CF. Resolution of pulsatile tinnitus after coil embolization of sigmoid sinus diverticulum. Austin J Cerebrovasc Dis 2014;1.

29 Hellstern V, Aguilar-Pérez M, Schob S, et al. Endovascular treatment of dural arteriovenous fistulas of the anterior or posterior condylar vein. Clin Neuroradiol 2019:29:341-9.

30 Brinjikji W, Lanzino G, Cloft HJ. Transvenous embolization of a posterior condylar canal fistula causing pulsatile tinnitus. J Neurointerv Surg 2021;13:680.

31 Eliezer M, Freitas RK, Fantoni M, et al. Selective embolization of the mastoid emissary vein for pulsatile tinnitus treatment: when is it indicated? I Neurointerv Surg 2020;12:999-1001.

32 Abdalkader M, Ma A, Cohen M, et al. Endovascular coiling of large mastoid emissary vein causing pulsatile tinnitus. Interv Neuroradiol 2020;26:821-5.
33 Rolffs J, Schmelzle R. Serious cerebral complications following unilateral ligation of the internal jugular vein. review of the literature and a case report. J Maxillofac Surg 1977:5:118-23.

34 Couloigner V, Grayeli AB, Bouccara D, et al. Surgical treatment of the high jugular bulb in patients with Ménière's disease and pulsatile tinnitus. Eur Arch Otorhinolaryngol 1999;256:224-9.

35 Newman CW, Jacobson GP, Spitzer JB. Development of the tinnitus handicap inventory. Arch Otolaryngol Head Neck Surg 1996;122:143-8.

36 Cognard C, Houdart E, Casasco A, et al. Long-term changes in intracranial dural arteriovenous fistulae leading to worsening in the type of venous drainage. Neuroradiology 1997;39:59-66.

37 Satomi J, van Dijk JMC, Terbrugge KG, et al. Benign cranial dural arteriovenous fistulas: outcome of conservative management based on the natural history of the lesion. J Neurosurg 2002;97:767-70.

38 Alexander MD, Halbach VV, Hallam DK, et al. Long-term outcomes of endovascular treatment of indirect carotid cavernous fistulae: superior efficacy, safety, and durability of transvenous coiling over other techniques. Neurosurgery 2019;85:E94-100.

39 Caton MT, Narsinh KH, Baker A, et al. Endovascular treatment strategy, technique, and outcomes for dural arteriovenous fistulas of the marginal sinus region. J Neurointerv Surg 2022;14:155-9.

40 Halbach VV, Higashida RT, Hieshima GB. Transvenous embolization of dural fistulas involving the cavernous sinus. Am I Neuroradiol 1989;10:377-83.

41 Cima RFF, Maes IH, Joore MA, et al. Specialised treatment based on cognitive behaviour therapy versus usual care for tinnitus: a randomised controlled trial. Lancet 2012;379:1951-9.

42 Hesser $\mathrm{H}$, Weise C, Westin VZ, et al. A systematic review and meta-analysis of randomized controlled trials of cognitive-behavioral therapy for tinnitus distress. Clin Psychol Rev 2011;31:545-53.

43 Westin VZ, Schulin M, Hesser H, et al. Acceptance and commitment therapy versus tinnitus retraining therapy in the treatment of tinnitus: a randomised controlled trial. Behav Res Ther 2011;49:737-47.

44 Philippot $\mathrm{P}, \mathrm{Nef} F$, Clauw $L$, et al. A randomized controlled trial of mindfulness-based cognitive therapy for treating tinnitus. Clin Psychol Psychother 2012;19:411-9.

45 Liang Z, Yang H, Cheng G, et al. Repetitive transcranial magnetic stimulation on chronic tinnitus: a systematic review and meta-analysis. BMC Psychiatry 2020;20:547.

46 Meng Z, Liu S, Zheng Y, et al. Repetitive transcranial magnetic stimulation for tinnitus. Cochrane Database Syst Rev 2011:CD007946.

47 Valluru K, Parkhill J, Gautam A, et al. Sound measurement in patient-specific 3D printed bench models of venous pulsatile tinnitus. Otol Neurotol 2020:41:e7-14. 